Document downloaded from:

http://hdl.handle.net/10251/109426

This paper must be cited as:

Mautschke, H.; Drache, F.; Senkovska, I.; Kaskel, S.; Llabrés I Xamena, FX. (2018).

Catalytic properties of pristine and defect-engineered Zr-MOF-808 metal organic frameworks. Catalysis Science \& Technology. 8(14):3610-3616. doi:10.1039/c8cy00742j

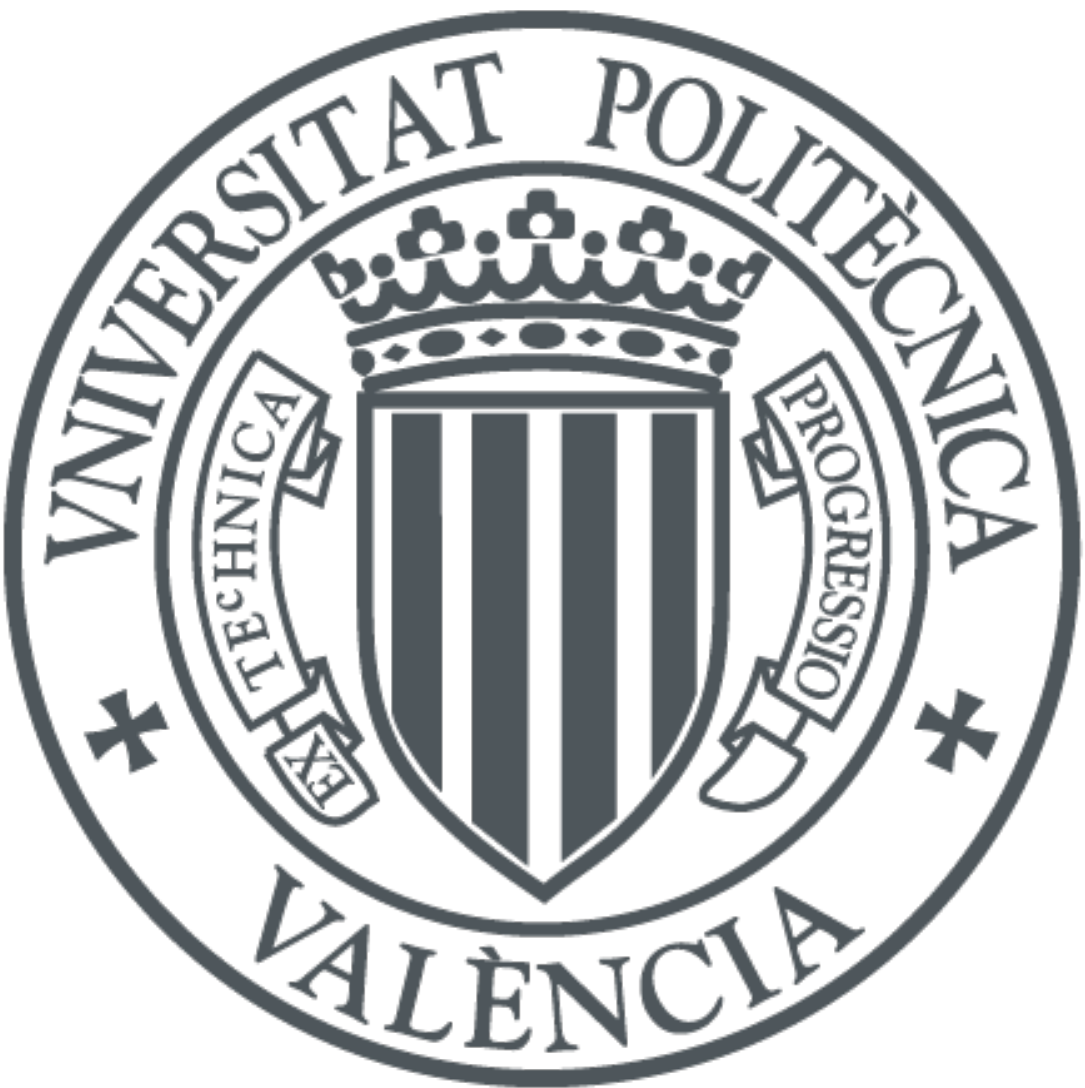

The final publication is available at

https://doi.org/10.1039/c8cy00742j

Copyright The Royal Society of Chemistry

Additional Information 


\title{
Catalytic properties of pristine and defect-engineered Zr-MOF-808 Metal Organic Frameworks
}

\section{H. -H. Mautschke, ${ }^{\text {a }}$ F. Drache, ${ }^{\text {b }}$ I. Senkovska, ${ }^{\text {b }}$ S. Kaskel ${ }^{\text {b }}$ and F. X. Llabrés i Xamena $^{\mathrm{a}^{*}}$}

a Instituto de Tecnología Química UPV-CSIC, Universitat Politècnica de València, Consejo Superior de Investigaciones Científicas, Avda. de los Naranjos s/n, 46022 Valencia, Spain

${ }^{\mathrm{b}}$ Department of Inorganic Chemistry, Dresden University of Technology, Bergstraße 66, 01062 Dresden, Germany

* Corresponding author: e-mail: fllabres@itq.upv.es

Fax: (+34) 963877809

\begin{abstract}
Various defect-engineered Zr-trimesate MOF-808 compounds (DE-MOF-808) have been prepared by mixing the tricarboxylate ligands with dicarboxylate ligands; viz. isophthalate, pyridine-3,5-dicarboxylate, 5-hydroxy-isophthalate, or 5-aminoisophthalate. The resulting mixed-ligand compounds, MOF-808-X (X = IP, Pydc, OH or $\mathrm{NH}_{2}$ ) were all found to be highly crystalline and isostructural to the unmodified MOF808. Pristine MOF-808 showed better catalytic performance than a UiO-66 reference compound for the Meerwein-Ponndorf-Verley (MPV) reduction of carbonyl compounds. This was attributed to a higher availability of coordinatively unsaturated $\mathrm{Zr}^{4+}$ sites (cus) in MOF-808 upon removal of formate ions. Meanwhile, cus in UiO-66 are only located at defect sites and are thus much less abundant. Further improvement of the catalytic activity of defect-engineered MOF-808-IP and MOF-808-Pydc was observed, which may be related with the occurrence of less crowded $\mathrm{Zr}^{4+}$ sites in DE-MOF-808. The wider pore structure of MOF-808 with respect to UiO-66 compounds translate into a sharp improvement of the activity for the MPV reduction of bulky substrates, as shown for estrone reduction to estradiol. Interestingly, MOF-808 produces a notable diastereoselectivity towards the elusive $17-\alpha$-hydroxy estradiol.
\end{abstract}

Keywords: Metal Organic Frameworks; heterogeneous catalysis; MOF-808; Zr-MOFs, Diastereoselective Meerwein-Pondorf-Verley; estrone reduction. 


\section{Introduction}

Metal Organic Frameworks (MOFs) are crystalline hybrid materials formed by metal ions (or oxoclusters) connected to polytopic organic molecules through coordination bonds forming extended mono-, bi- or tridimensional networks defining a strictly regular system of pores and cavities of molecular dimensions. ${ }^{1}$ The huge variability of chemical compositions and pore architectures, the large surface areas and pore volumes attainable with these materials, and the possibility to introduce new functionalities in preformed MOFs through post-synthesis methods, ${ }^{2,3}$ all make MOFs excellent candidates for a number of technological applications, including heterogeneous catalysis. ${ }^{4-7}$ In this sense, the $\mathrm{Zr}$-containing terephthalate known as $\mathrm{UiO}-66^{8}$ and its derivatives have attracted a great deal of attention in recent years. ${ }^{9-13}$ These compounds are formed by hexameric $\left[\mathrm{Zr}_{6} \mathrm{O}_{4}(\mathrm{OH})_{4}\right]$ octahedral oxoclusters connected by 12 terephthalate linkers into a face centered cubic packing. The high coordination number of the inorganic building units endows the material with a remarkable thermal, chemical and mechanical stability, ${ }^{14}$ which is seldom found in many known MOF compounds. Together with this high stability, an attractive characteristic of UiO-66-type compounds is the possibility to prepare a battery of derivatives by introducing additional functional groups in the terephathalate linkers ${ }^{15-17}$ and to prepare expanded versions of UiO-66 by replacing terephthalate by longer linear dicarboxylate linkers (viz., UiO-67, UiO-68 and related compounds ${ }^{18}$ ). However, the coordination sphere of $\mathrm{Zr}^{4+}$ ions in UiO-66 is completely blocked by the carboxylate linkers, thus lacking coordination vacancies available for substrate binding. Therefore, UiO-66 materials rely on the formation of linker defects (either intrinsic or intentional) to display the reactivity typical of solid acid catalysts. ${ }^{9}, 19$ In this way, it is well know that a direct correlation exist between the number of missing linker defects and the catalytic activity of UiO-66. ${ }^{9}$ A second limitation of UiO-66 is that it features a relatively narrow pore system, accessible through windows of about $6 \AA{ }^{8}$ which largely delimits the substrates that can reach the active sites located at the internal surface, and thus, the reactivity scope of this material.

Motivated by the excellent catalytic properties displayed by UiO-66-type materials, and in an attempt to overcome the two limitations mentioned above (viz., lack of coordination vacancies and narrow pore system), we have addressed our efforts to other $\mathrm{Zr}$-containing compounds. Among several possible candidates, we have found that $\mathrm{Zr}$ trimesate known as MOF- $808^{20}$ can be an interesting alternative with a high potential for catalytic applications. This compound has similar $\mathrm{Zr}_{6}$-oxoaggregates than those present 
in UiO-66, forming a tridimensional network with cavities of $18.4 \AA$ and apertures of 14 $\AA$ (see Fig. 1). In the MOF-808 structure, each cluster is connected by 6 trimesate linkers, while the other coordination positions of $\mathrm{Zr}$ ions are saturated by bridging formate molecules. These formate molecules can be removed by simple solvent washing or mild thermal treatment, thus leaving two coordination vacancies on each metal site.

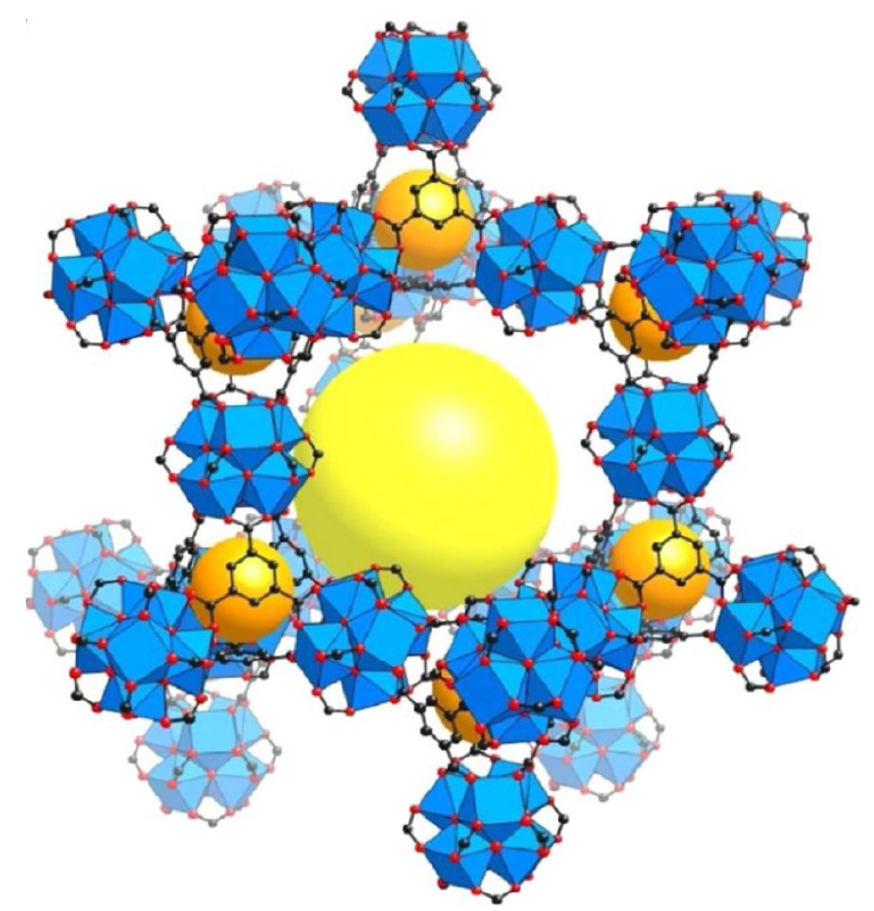

Fig 1. Structure of MOF-808, showing the $\mathrm{Zr}_{6}$ oxoaggregates and evidencing the large adamantane-like cavities. $\mathrm{C}$, black; $\mathrm{O}$, red; $\mathrm{Zr}$, blue polyhedra. $\mathrm{H}$ atoms are omitted for clarity. Yellow and orange balls indicate the space in the framework. Reproduced with permission from Ref. ${ }^{20}$. Copyright 2014 American Chemical Society.

Besides MOF-808, other wide pore compounds containing similar $\mathrm{Zr}_{6}$ oxoaggregates with open metal sites might be considered as well, ${ }^{18}$ such as NU-1000, ${ }^{21} \mathrm{PCN}-222,{ }^{22}$ PCN-700, ${ }^{23}$ DUT-51, ${ }^{24}$ or the PIZOFs. ${ }^{25}$ However, a clear drawback of these compounds is that they usually require the use of non-commercial and/or expensive organic ligands, which introduce additional synthesis steps and rise up the final price, thus hampering the large scale synthesis of these MOFs. This is in sharp contrast with readily available and cheap trimesic acid used in the synthesis of MOF-808.

In order to increase further the availability of the $\mathrm{Zr}$ sites in MOF-808, we have prepared defect-engineered MOF-808 materials (DE-MOF) by a mixed-ligand approach (see Scheme 1). Thus, we have combined the tritopic trimesic acid ligands (1) with a small amount (ca. 10\%) of a ditopic ligand, such as isophtalic acid (2), pyridine-3,5- 
dicarboxylic acid (3), or 5-hydroxy- (4) and 5-amino-isophthalic acid (5) to prepare a series of mixed-ligand MOFs. A similar strategy has been shown to increase the number of open metal sites in related $\mathrm{Cu}^{26}$ and $\mathrm{Ru}^{27,28}$ trimesate compounds, which translated into a considerable improvement of the catalytic properties of the DE-MOF with respect to the pristine compound.

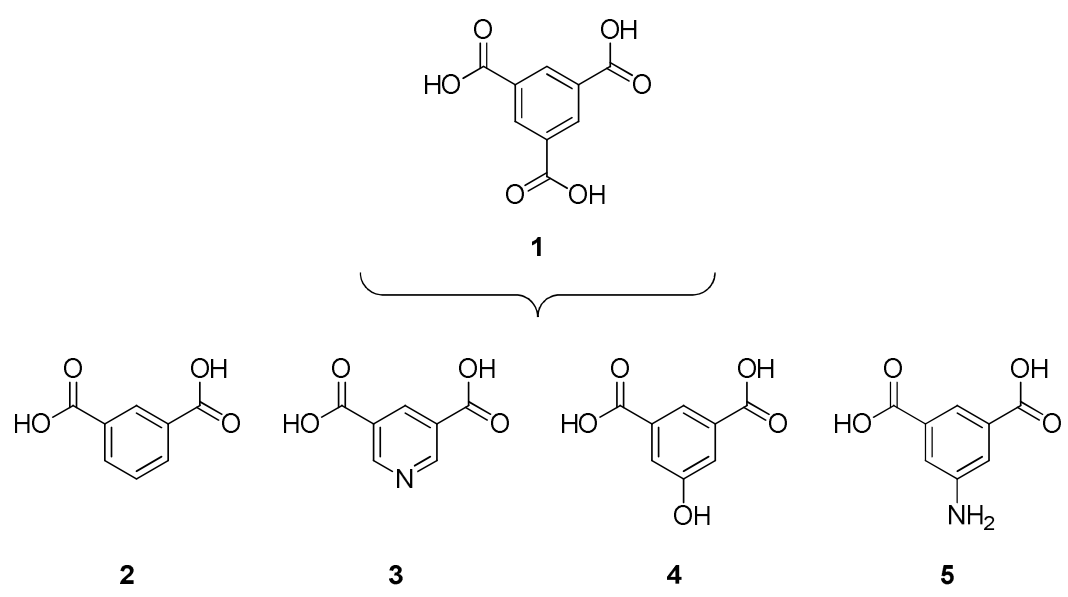

Scheme 1. Trimesate $(\mathbf{1})$ is combined with various ditopic ligands (2-5) to prepare defectengineered MOF-808, DE-MOFs.

Herein, we have investigated the catalytic properties of both, pristine and DE-MOF808 compounds, and the results have been compared with those obtained over UiO-66 as a reference compound. As a test reaction, we have considered the Meerwein-PonndorfVerley reduction of carbonyl compounds using an alcohol as reducing agent. Indeed, de Vos and co-workers have recently shown that MOF-808 can efficiently catalyse this reaction. ${ }^{29}$ The benefits of MOF-808 having a wider pore system and a higher concentration of coordinatively unsaturated $\mathrm{Zr}^{4+}$ sites (cus), as compared with UiO-66, are evidenced by studying the reduction of ketones of different size.

\section{Experimental section}

\section{Synthesis of pristine and DE-MOFs}

MOF-808: Pristine zirconium MOF-808 material was prepared with slightly modifications from an earlier reported procedure by Furukawa et. al. ${ }^{20}$ Briefly, a solution was prepared containing 242,5 $\mathrm{mg}$ of $\mathrm{ZrOCl}_{2} * 8 \mathrm{H}_{2} \mathrm{O}(0,75 \mathrm{mmol}), 105 \mathrm{mg}$ of trimesic acid $(0,5 \mathrm{mmol})$ and $22,5 \mathrm{~mL}$ of a $\mathrm{DMF} / \mathrm{HCO}_{2} \mathrm{H} 1: 1$ (v/v) mixture. The solution was transferred into a Teflon lined autoclave and heated inside an oven at $130{ }^{\circ} \mathrm{C}$ for $48 \mathrm{~h}$. After cooling down to room temperature, the material was recovered by centrifugation 
and washed for 3 days with DMF (changing the solvent 2 times per day) and for another 3 days with EtOH (changing the solvent 2 times per day). After removing the solvent by centrifugation, the solid was dried in air. X-ray diffraction (PhillipsX'Pert, $\mathrm{Cu} \mathrm{K \alpha}$ radiation) was used to confirm the expected structure type and high crystallinity of the material.

DE-MOF-808: DE-MOF-808 materials were prepared by replacing $10 \mathrm{~mol} \%$ of trimesic acid by equimolar amounts of defective linker: a) isophthalic acid $(8,3 \mathrm{mg}, 0,05 \mathrm{mmol})$ for MOF-808-IP, b) 3,5-pyridinedicarboxylic acid (8,4 mg, 0,05 mmol) for MOF-808Pydc, c) 5-hydroxyisophthalic acid $(9,1 \mathrm{mg}, 0,05 \mathrm{mmol})$ for MOF-808-OH and d) 5aminoisophthalic acid $(9,1 \mathrm{mg}, 0,05 \mathrm{mmol})$ for MOF-808- $\mathrm{NH}_{2}$. The remaining synthesis steps were kept the same as for the pristine MOF-808 material.

UiO-66: The reference zirconium terephthalate UiO-66 material was prepared according to the reported procedure. ${ }^{16}$ Briefly, $750 \mathrm{mg}$ of $\mathrm{ZrCl}_{4}$ and $740 \mathrm{mg}$ of terephthalic acid were dissolved in $90 \mathrm{~mL}$ of DMF (Zr:ligand:DMF molar ratio of 1:1:220) and the solution was kept in a closed round bottom flask at $80^{\circ} \mathrm{C}$ in an oil heating bath for $12 \mathrm{~h}$ without stirring, followed by another $24 \mathrm{~h}$ at $100^{\circ} \mathrm{C}$. The resulting material was recovered by filtration and washed thoroughly with fresh DMF. Then the solid was washed three times by soaking in dichloromethane for $3 \mathrm{~h}$. Finally, the solid was recovered by filtration and dried under vacuum. The amount of missing linker defects in this UiO-66 sample was estimated to be $c a$. $7 \%$ from the corresponding TGA curve, following the method proposed by Valenzano et. al. ${ }^{30}$

DUT-67: $\mathrm{ZrCl}_{4}(1.38 \mathrm{~g}, 6 \mathrm{mmol})$ was dissolved in $150 \mathrm{~mL}$ of $\mathrm{N}, \mathrm{N}$-dimethylformamide $/ N$ methyl-2-pyrrolidone mixture (DMF/NMP, 1:1) by ultrasonication for 10 minutes. Afterwards, 2,5-thiophenedicarboxylic acid (0.66 g, $4 \mathrm{mmol})$ was added and the mixture was sonicated again for 5 minutes. Then formic acid $(26.8 \mathrm{~mL}, 120 \mathrm{eq})$ was added and the resulting mixture was divided in to 3 parts and distributed between three Schott flasks (500 mL each) and placed into an oven for $72 \mathrm{~h}$ at $85{ }^{\circ} \mathrm{C}$. The white precipitate was filtered off and washed several times with DMF.

\section{Results and discussion}

\section{Synthesis and characterization of pristine and DE-MOF-808}

Pristine MOF-808 was prepared following the procedure reported by Furukawa et al. ${ }^{20}$ Slightly modified synthesis were used to prepare the mixed-ligand, defect engineered MOFs, by replacing $10 \%$ of the trimesate ligands by the defect-inducing ditopic ligands, 
as described in detail in the Experimental section. These compounds will be hereafter referred to as MOF-808-X; where $\mathrm{X}=\mathrm{IP}$ (isophthalate), Pydc (pyridine-3,5dicarboxylate), $\mathrm{OH}$ (5-hydroxy-isophthalate), or $\mathrm{NH}_{2}$ (5-amino-isophthalate).

According to the powder X-ray diffraction patterns shown in Fig. 2, all the materials were found to be isostructural and highly crystalline. All the diffraction peaks can be assigned to the expected structure for MOF-808 (space group $F \mathrm{~d}-3 m$, spn topology). ${ }^{1} \mathrm{H}$ NMR of the digested solids (see Fig.S1 in the ESI) confirmed the incorporation of the ditopic ligands. Characteristic peaks of the ligands were used to determine the amount of dicarboxylate ligands incorporated in each case, as summarized in Table S1 (ESI). Incorporation of the defective ligands was found to be very low in all cases (ca. $3-7 \%)$. It was not possible to increase these values even when the amount of defective ligand introduced in the starting reaction mixture was increased from $10 \%$ to $20 \%$. As a consequence, no significant differences were observed in the FTIR or TGA curves of pristine and DE-MOF compounds. Meanwhile, introduction of ditopic ligands in MOF808 produced only minor changes on the textural properties of MOF-808, as summarized in Table S2 (ESI). For instance, $\mathrm{S}_{\mathrm{BET}}$ and pore volume varied from $1345 \mathrm{~m}^{2} \mathrm{~g}^{-1}$ and 0.60 $\mathrm{cm}^{3} \mathrm{~g}^{-1}$ for pristine MOF-808, to $1592 \mathrm{~m}^{2} \mathrm{~g}^{-1}$ and $0.74 \mathrm{~cm}^{3} \mathrm{~g}^{-1}$ for the most porous sample, MOF-808-Pydc.

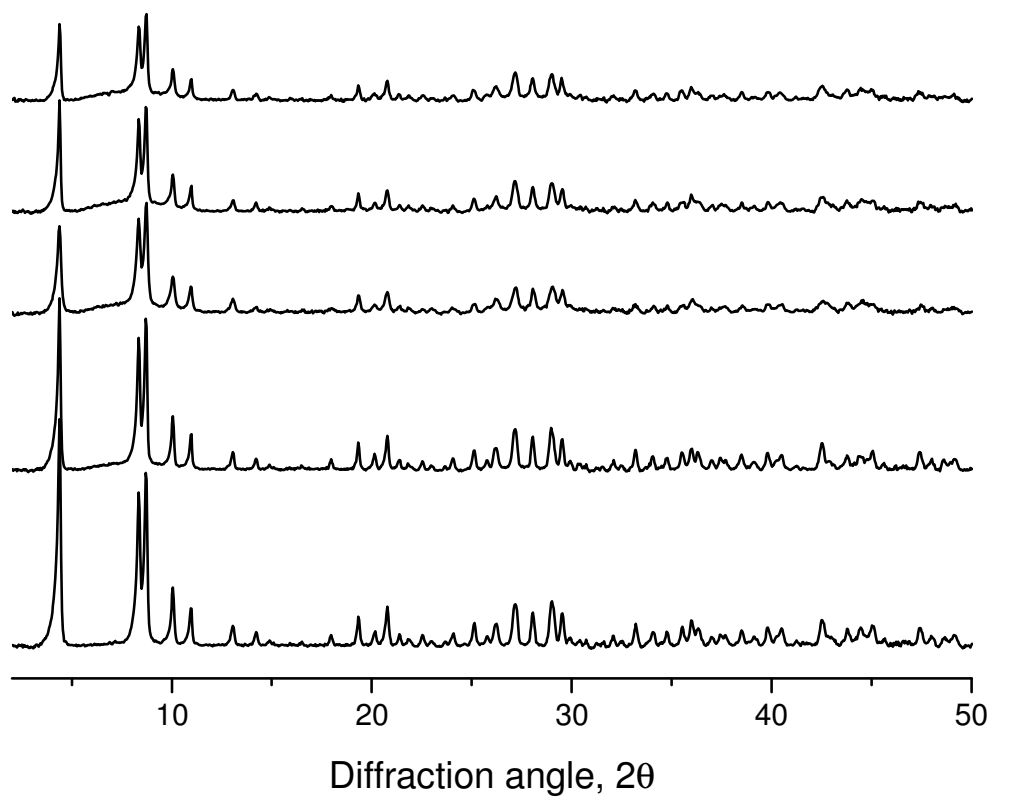

Fig. 2. X-ray diffraction patterns ( $\mathrm{Cu} \mathrm{K} \alpha$ radiation) of (from bottom to top): MOF-808, MOF-808-IP, MOF-808-Pydc, MOF-808-OH and MOF-808-NH2. 


\section{Catalytic properties of pristine and DE-MOF-808}

Meerwein-Ponndorf-Verley reduction of cyclohexanone. The catalytic activity of pristine and DE-MOFs for the MPV reduction of carbonyl compounds was first evaluated using cyclohexanone as a model compound and isopropanol as both reducing agent and solvent. The results were then compared with a reference UiO-66 material containing $c a$. $7 \%$ of linker defects measured under the same reaction conditions. Fig. 3 and Table 1 summarize the results obtained.

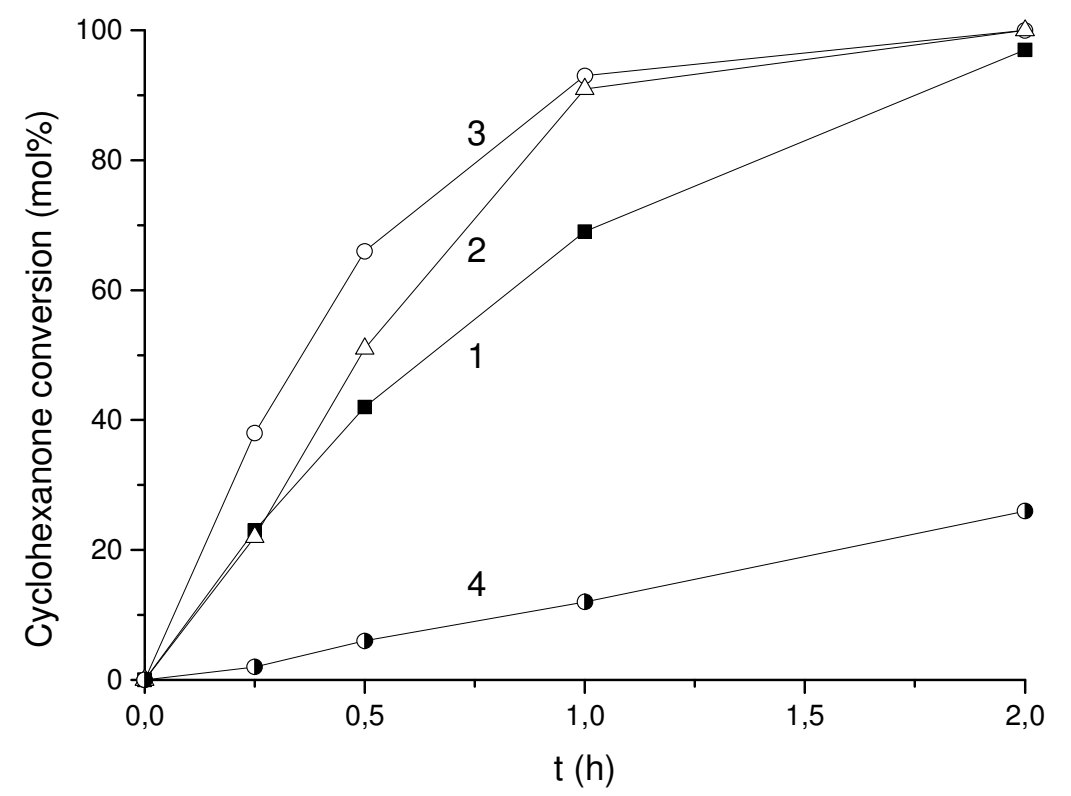

Fig. 3. Conversion of cyclohexanone over: MOF-808 (curve 1), MOF-808-IP (curve 2), MOF-808-Pydc (curve 3), and a reference UiO-66 containing ca. 7\% missing linker defects (curve 4).

As it can be seen in Fig. 3, conversion of cyclohexanone is almost complete after $2 \mathrm{~h}$ using the pristine MOF-808 catalyst. The reaction was found to be fully selective, being cyclohexanol the only product detected in all cases. MOF-808 was found to be much more active than the reference UiO-66 compound. Thus for instance, cyclohexanone conversion after $1 \mathrm{~h}$ was $69 \%$ and $12 \%$ over MOF-808 and UiO-66, respectively (compare entries 1 and 3 in Table 1). In our opinion, this difference in activity shall not be attributed to eventual diffusion problems in the case of UiO-66, since cyclohexanone is small enough to enter the pores of UiO-66 (see Fig. S2 in ESI). Indeed, full cyclohexanone conversion is slowly attained after $c a$. 24 h over UiO-66 (Fig. S3 in ESI). Moreover, SEM revealed that both UiO-66 and MOF-808 have similar particle size (see Fig. S4 in ESI), so that no significant differences are expected in the amount of sites 
exposed at the external surface of the crystallites. Rather, the difference in reactivity between MOF-808 and UiO-66 is most likely due to the higher concentration of $\mathrm{Zr}$ coordinatively unsaturated metal sites in the former material, created upon removal of the formate anions during catalyst activation. As we have mentioned above, UiO-66 lacks such open metal sites, and the only coordination positions available are those associated with missing linkers. Note that, according to the model suggested in ref. ${ }^{9}$ for missing linker defects, an UiO-66 sample with $7 \%$ of missing linkers defects contains only $14 \%$ of the total $\mathrm{Zr}$ ions exposed and available for the reaction (each missing linker molecule creates two open metal sites, one in each of two neighbour oxoaggregates). In contrast, all $\mathrm{Zr}$ ions are accessible in MOF-808 after removal of the formate ligands.

To complete our study, and in order to lend further support to our conclusion that the observed differences in catalytic activity are related directly with the relative amount of available active sites, we have included a third $\mathrm{Zr}$-containing MOF in the present study, DUT-67. ${ }^{31}$ In this compound, the $\mathrm{Zr}_{6}$ clusters are connected to 8 carboxylate ligands, thus lying between MOF-808 (6-connected) and UiO-66 (12-connected). As expected, the rate of cyclohexanone reduction is also intermediate between that observed for MOF-808 and UiO-66 (see Fig. S3 in ESI).

Table 1. MPV reduction of cylohexanone over various Zr-MOFs. ${ }^{a}$

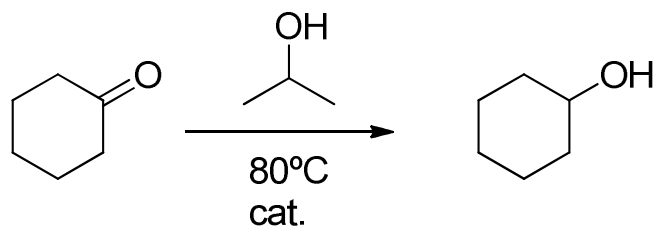

\begin{tabular}{llcc}
\hline Entry & Catalyst & Time $(\mathbf{h})$ & Conv $^{(\mathbf{m o l} \%)^{b}}$ \\
\hline 1 & UiO-66 & 1 & 12 \\
2 & & 24 & $>99$ \\
3 & MOF-808 & 1 & 69 \\
4 & & 2 & 97 \\
5 & MOF-808-IP & 1 & 91 \\
6 & & 2 & $>99$ \\
7 & MOF-808-Pydc & 1 & 93 \\
8 & & 2 & $>99$
\end{tabular}

${ }^{a}$ Reaction conditions: $10 \mathrm{mg}$ cyclohexanone $(0.1 \mathrm{mmol}),{ }^{\mathrm{i}} \mathrm{PrOH}(0.5 \mathrm{~mL}, c a .6 .5 \mathrm{eq})$ and $\mathrm{Zr}-\mathrm{MOF}$ (5 mg, ca. $14 \mathrm{~mol} \% \mathrm{Zr}$ ), 80 ${ }^{\circ} \mathrm{C} .{ }^{b}$ Conversion, determined by GC. Selectivity to cyclohexanol was $>99 \%$ in all cases. 
Interestingly, the use of DE-MOFs, MOF-808-IP and MOF-808-Pydc, containing defect-inducing linkers, brings about a marked increase of the catalytic activity compared with the pristine MOF-808 (see Fig. 3). Thus, the yield of cyclohexanol obtained after 1 $\mathrm{h}$ increased from 69\% (MOF-808, entry 3) to 91\% and 93\% (MOF-808-IP and MOF-808Pydc, entries 5 and 7, respectively). FTIR spectroscopy of adsorbed CO at $77 \mathrm{~K}$ (see Fig $\mathrm{S} 5$ in ESI) revealed that both, the concentration and relative acid strength of the $\mathrm{Zr}^{4+}$ open metal sites are virtually identical in pristine MOF-808 and MOF-808-Pydc compounds. Therefore, we attribute the observed increment of the reactivity of DE-MOFs with respect to the pristine material to the creation of additional coordination vacancies on the already exposed $\mathrm{Zr}$ sites due to the replacement of tritopic trimesate ligands by the ditopic ligands, thus leaving less sterically crowded Zr active sites (see Fig. S6 in ESI). In this sense, it is important to stress that the generally accepted mechanism for the MPV reduction of ketones over Lewis acid catalysts assumes the simultaneous adsorption of the alcohol and the ketone on the active site and the direct hydrogen transfer from the alcohol to the ketone, involving a six-membered cyclic transition state, as shown in Scheme 2. ${ }^{32}$ Therefore, it seems reasonable to hypothesize that less shielded $\mathrm{Zr}$ acid sites will form such a transition state more easily. Further computational studies are currently underway to evaluate this hypothesis in more detail.

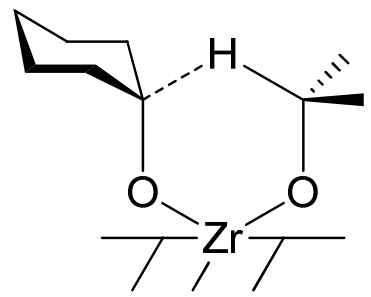

MOF

Scheme 2. Proposed transition state for the MPV reduction of cyclohexanone with isopropanol over Zr-containing MOF-808 compounds.

In the case of MOF-808-OH and MOF-808- $\mathrm{NH}_{2}$, no significant improvement was observed with respect to pristine MOF-808 (see Fig. S7 in ESI). This probably indicates that the presence of $-\mathrm{OH}$ and $-\mathrm{NH}_{2}$ groups in position 5 of the ligands introduces further steric hindrance around the $\mathrm{Zr}$ sites, similar to what happens in pristine MOF-808, thereby precluding any improvement of the catalytic activity with respect to the parent material.

Finally, it is important to point out that all the materials were found to be stable under the reactions conditions used, and no significant differences were observed in the corresponding XRDs of the materials after reaction (see Fig. S8 in ESI). Consequently, 
no significant decrease of the catalytic activity was observed upon reuse of the material for at least 4 consecutive cycles.

Meerwein-Ponndorf-Verley reduction of estrone (E1). As we have mentioned in the Introduction, MOF-808 has a wider pore system with respect to UiO-66, with pore entries of about $14 \AA$ and $6 \AA$, respectively. From the catalytic point of view, this is a clear advantage when dealing with the conversion of bulky substrates (with a size comprised between 6 and $14 \AA$ ), which will only react at the external surface of UiO-66 but will freely diffuse inside the pores of MOF-808, thus reaching all the active sites.

In order to evaluate the benefits of a wide-pore catalyst such as MOF-808 as compared with UiO-66, we have studied the MPV reduction of a bulky ketone: estrone (E1, approximate dimensions: $11.2 \AA$ x $6.2 \AA$ x $4.2 \AA$, as extracted from the optimized structure using MOPAC2016, ${ }^{33}$ see Fig. S9 in ESI). A summary of the results obtained with various Zr-MOF catalysts is shown in Table 2.

Table 2. MPV reduction of estrone over various $\mathrm{Zr}-\mathrm{MOFs}{ }^{a}$

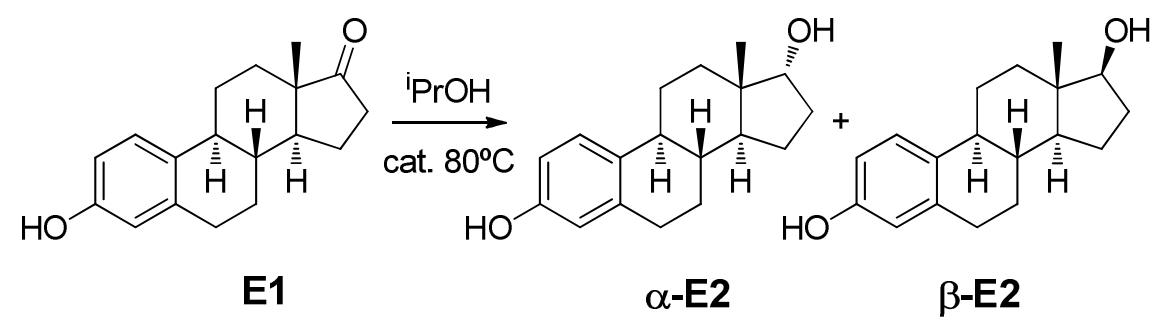

\begin{tabular}{llccc}
\hline Entry & Catalyst & Time $(\mathbf{h})$ & Conv $_{(\mathbf{m o l} \%)^{\boldsymbol{b}}}$ & d.r. $^{\boldsymbol{c}}$ \\
\hline 1 & UiO-66 & 48 & 2 & n.d. \\
2 & MOF-808 & 4 & 24 & \\
3 & & 24 & 87 & $40: 60$ \\
4 & MOF-808-Pydc & 4 & 61 & \\
5 & & 24 & 91 & $38: 62$
\end{tabular}

${ }^{a}$ Reaction conditions: $20 \mathrm{mg}$ estrone $(0.08 \mathrm{mmol}),{ }^{\mathrm{i}} \mathrm{PrOH}(1 \mathrm{~mL}, c a .16 \mathrm{eq})$ and $\mathrm{Zr}-\mathrm{MOF}$ (5 mg, ca. $18 \mathrm{~mol} \% \mathrm{Zr}$ ), $80^{\circ} \mathrm{C} .{ }^{b}$ Conversion. Determined by GC. Estradiols were the only product detected. ${ }^{c} \alpha-\mathrm{E} 2: \beta-\mathrm{E} 2$ diastereomeric ratio, calculated from the ${ }^{1} \mathrm{H}$ NMR spectra of the reaction filtrates (for the exact procedure used, see Fig.S10 in ESI).

Under the experimental conditions used, UiO-66 was found to be almost inactive for the reduction of estrone (only $2 \%$ conversion after $48 \mathrm{~h}$ ). This is most likely due to a size- 
exclusion effect, which prevents the large estrone molecule to cross the $\sim 6 \AA$ triangular pores to reach the active sites located (mostly) at the internal surface of UiO-66. On the contrary, almost full estrone conversion was attained over the wide pore MOF-808 materials after $24 \mathrm{~h}$ of reaction, with full selectivity to the expected estradiol (E2). As it was the case of cyclohexanone reduction, the introduction of defective-inducing pyridinedicarboxylate ligands was found to be beneficial for the catalytic activity. Thus, the conversion obtained after $4 \mathrm{~h}$ of reaction was $24 \%$ and $61 \%$, respectively for MOF808 and MOF-808-Pydc. However, the catalytic performance of MOF-808-IP was very similar to that of the pristine MOF-808: maximum yield of $78 \%$ after $24 \mathrm{~h}$. This lower activity with respect to MOF-808-Pydc might be related to a faster deactivation associated to product adsorption, although more experiments would be necessary to reach a more satisfactory explanation.

In general, the synthesis of $17 \alpha$-hydroxy steroids (such as $\boldsymbol{\alpha}$-E2) by reduction of the corresponding 17-oxo compound is challenging. Usually, the $17 \beta$ isomer is obtained almost exclusively when $\mathrm{NaBH}_{4}$ or other reducing agents are employed, due to the steric hindrance imposed by the 18-methyl group on the approaching direction of the reducing agent. Additional reactions are then required to produce the inversion of the $17 \beta$-hydroxy steroids to the $17 \alpha$ compounds through Mitsunobu reactions, ${ }^{34}$ which involve multiple protection-deprotection steps. And even then, the yields and selectivities to the $17 \alpha-\mathrm{OH}$ steroids are only moderate at best. For example, Han et al. reported on the synthesis of a related $17 \alpha-\mathrm{OH}$ steroid ( $5 \alpha$-androstane-3 $\beta, 17 \alpha$-diol) from the 17 -oxo compound by a 4 steps reaction involving reduction, tosylation, acetate substitution and hydrolysis, with a final yield of the $17 \alpha$ product as low as $4 \% .{ }^{35}$ Ohta et al. reported later a modification of this procedure by adding three additional steps: epoxidation with peracetic acid, reduction with $\mathrm{NaBH}_{4}$ and hydrolysis with $\mathrm{NaOH}$. The reported final yield of the $17 \alpha-\mathrm{OH}$ compound was $54 \% .{ }^{36}$ Göndös and Orr followed a different approach to $17 \alpha-\mathrm{OH}$ steroid compounds by using a chiral Rh complex as stereoselective reducing agent, yielding the $17 \alpha$-hydroxy-estrone-3-methyl ether in $36 \%$, together with the $17 \beta$-compound in $26 \%$ yield. ${ }^{37}$

Given the elusive character of the $17 \alpha$-hydroxy steroid derivatives, it is evident that development of alternative preparative methods would be highly desirable. In this sense, it would be very convenient to use easily recoverable (and cheap) solid catalysts, along 
with the design of single step reaction processes and minimization of waste generation and use of additional reagents.

Therefore, given the well known high chemo-, regio- and stereoselectivity attainable with MPV reactions, ${ }^{32}$ we have evaluated the diastereomeric ratio between alpha- and beta-estradiol ( $\boldsymbol{\alpha}$-E2 and $\boldsymbol{\beta}$-E2) obtained using both MOF-808 and MOF-808-Pydc as catalysts. Interestingly, we found that both materials afforded a mixture of the two alcohols in a $c a .40: 60 \alpha: \beta$ ratio, which translates into an overall yield of $c a .35 \%$ of the $\boldsymbol{\alpha}$-E2 isomer after $24 \mathrm{~h}$ of reaction time. This finding is most probably related with the relative steric hindrance of the two transition states leading to $\alpha$-E2 and $\beta$-E2 products inside the restricted space of the MOF cavities, similar to what was already pointed out for MPV reactions taking place inside the pores of zeolites. ${ }^{38,} 39$ Although the stereoselectity attained with MOF-808 compounds is still far from optimal, it is worth mentioning that $17 \alpha$-hydroxy-estradiol was cleanly obtained directly from estrone with moderate yields in a single reaction step, using only isopropanol as the sole reagent and avoiding any additional protection/deprotection steps. Isolation of pure $\alpha$ and $\beta$ isomers from the reaction filtrate can be readily achieved by TLC or column purification without any further workout.

\section{Conclusions}

Herein we have reported on the synthesis of various defect-engineered MOF-808 materials by mixing the tricarboxylate trimesate ligands of MOF-808 with dicarboxylate ligands; viz. isophthalate (IP), pyridine-3,5-dicarboxylate (Pydc), 5-hydroxy-isophthalate $(\mathrm{OH})$, or 5-amino-isophthalate $\left(\mathrm{NH}_{2}\right)$. The resulting mixed-ligand compounds, MOF$808-\mathrm{X}\left(\mathrm{X}=\mathrm{IP}, \mathrm{Pydc}, \mathrm{OH}\right.$ or $\left.\mathrm{NH}_{2}\right)$ were all found to be highly crystalline and isostructural to the unmodified MOF-808.

The catalytic activity of pristine and mixed-ligand MOF-808 compounds have been evaluated for the Meerwein-Ponndorf-Verley (MPV) reduction of carbonyl compounds using isopropanol as reducing agent, and the results were compared with a reference UiO66 material containing $c a$. $7 \%$ of linker defects. When a small molecule such as cyclohexanone was used as substrate, MOF-808 was found to be more active than UiO66. The higher activity of MOF-808 with respect to UiO-66 was attributed to the fact that in MOF-808, all $\mathrm{Zr}^{4+}$ ions are accessible and can participate in the reaction (once the bridging formate ions are removed). Meanwhile, the only accessible acid sites in UiO-66 
are those associated with linker defects (which represent only $14 \%$ of the total $\mathrm{Zr}$ ions of the solid). Partial replacement of trimesate linkers in MOF-808 by ditopic ligands results in a further improvement of the catalytic performance with respect to the pristine MOF808. This is probably related to the creation of less hindered $\mathrm{Zr}$ open metal sites when dicarboxylate ligands are used, which facilitate the formation of the 6-membered cyclic transition state (see Scheme 2). The presence of wide pores in MOF-808 with respect to UiO-66 translates into a significant improvement of the catalytic activity for converting bulky substrates, as it is shown here for the reduction of estrone to estradiol. Interestingly, the process produces reasonable amounts of the elusive $17 \alpha$-hydroxy estradiol in a single reaction step.

\section{Conflicts of interest}

There are no conflicts of interest to declare.

\section{Acknowledgements}

This project has received funding from the European Union's Horizon 2020 research and innovation programme under the Marie Sklodowska-Curie grant agreement No. 641887 (project acronym:DEFNET). Financial support from the Spanish Ministry of Economy and Competitiveness (program Severo Ochoa SEV20120267), the Spanish Ministry of Science and Innovation (project MAT2014-52085-C2-1-P), and the German Research Foundation (project KA 1698/19-1) is also gratefully acknowledged. The Microscopy Service of the Universitat Politècnica de València are gratefully acknowledged for the SEM images.

\section{References}

1. J. L. C. Rowsell and O. M. Yaghi, Microporous Mesoporous Mater., 2004, 73, 3-14.

2. Z. Q. Wang and S. M. Cohen, Chem. Soc. Rev., 2009, 38, 1315-1329.

3. A. D. Burrows, in Metal Organic Frameworks as Heterogeneous Catalysts, eds. F. X. Llabrés i Xamena and J. Gascon, The Royal Society of Chemistry, Cambridge, 2013, ch. 3, pp. 31-75.

4. S. M. J. Rogge, A. Bavykina, J. Hajek, H. Garcia, A. I. Olivos-Suarez, A. SepúlvedaEscribano, A. Vimont, G. Clet, P. Bazin, F. Kapteijn, M. Daturi, E. V. Ramos Fernandez, F. X. Llabrés i Xamena, V. Van Speybroeck and J. Gascon, Chem. Soc. Rev., 2017, 46, 31343184.

5. J. Gascon, A. Corma, F. Kapteijn and F. X. Llabrés i Xamena, ACS Catal., 2014, 4, 361378. 
6. A. Corma, H. Garcia and F. X. Llabrés i Xamena, Chem. Rev., 2010, 110, 4606-4655.

7. J. Y. Lee, O. K. Farha, J. Roberts, K. A. Scheidt, S. T. Nguyen and J. T. Hupp, Chem. Soc. Rev., 2009, 38, 1450-1459.

8. J. H. Cavka, S. Jakobsen, U. Olsbye, N. Guillou, C. Lamberti, S. Bordiga and K. P. Lillerud, J. Am. Chem. Soc., 2008, 130, 13850-13851.

9. F. G. Cirujano, A. Corma and F. X. Llabrés i Xamena, Chem. Eng. Sci., 2015, 124, 52-60.

10. V. L. Rechac, F. G. Cirujano, A. Corma and F. X. Llabrés i Xamena, Eur. J. Inorg. Chem., 2016, 2016, 4512-4516.

11. C. Rösler, S. Dissegna, V. L. Rechac, M. Kauer, P. Guo, S. Turner, K. Ollegott, H. Kobayashi, J. Yamamoto, D. Peeters, Y. Wang, S. Matsumura, G. Van Tendeloo, H. Kitagawa, M. Muhler, F. X. Llabrés i Xamena and R. A. Fischer, Chem. Eur. J, 2017, 23, 3583-3594.

12. F. Vermoortele, R. Ameloot, A. Vimont, C. Serre and D. De Vos, Chem. Commun., 2011, 47, 1521-1523.

13. F. Vermoortele, M. Vandichel, B. Van de Voorde, R. Ameloot, M. Waroquier, V. Van Speybroeck and D. E. de Vos, Angew. Chem., Int. Ed., 2012, 51, 4887-4890.

14. H. Wu, T. Yildirim and W. Zhou, J. Phys. Chem. Lett., 2013, 4, 925-930.

15. S. J. Garibay and S. M. Cohen, Chem. Commun., 2010, 46, 7700-7702.

16. M. Kandiah, M. H. Nilsen, S. Usseglio, S. Jakobsen, U. Olsbye, M. Tilset, C. Larabi, E. A. Quadrelli, F. Bonino and K. P. Lillerud, Chem. Mater., 2010, 22, 6632-6640.

17. M. Kandiah, S. Usseglio, S. Svelle, U. Olsbye, K. P. Lillerud and M. Tilset, J. Mater. Chem., 2010, 20, 9848-9851.

18. Y. Bai, Y. Dou, L. H. Xie, W. Rutledge, J. R. Li and H. C. Zhou, Chem. Soc. Rev., 2016, 45, 2327-2367.

19. F. Vermoortele, B. Bueken, G. Le Bars, B. Van de Voorde, M. Vandichel, K. Houthoofd, A. Vimont, M. Daturi, M. Waroquier, V. Van Speybroeck and D. E. de Vos, J. Am. Chem. Soc., 2013, 135, 11465-11468.

20. H. Furukawa, F. Gandara, Y. B. Zhang, J. Jiang, W. L. Queen, M. R. Hudson and O. M. Yaghi, J. Am. Chem. Soc., 2014, 136, 4369-4381.

21. J. E. B. Mondloch, W., D. Fairen-Jimenez, S. Kwon, E. J. DeMarco, M. H. Weston, A. A. Sarjeant, S. T. Nguyen, P. C. Stair, R. Q. Snurr, O. K. Farha and J. T. Hupp, J. Am. Chem. Soc., 2013, 135, 10294-10297.

22. D. Feng, Z. Y. Gu, Y. P. Chen, J. Park, Z. Wei, Y. Sun, M. Bosch, S. Yuan and H. C. Zhou, J. Am. Chem. Soc., 2014, 136, 17714-17717.

23. S. Yuan, W. Lu, Y.-P. Chen, Q. Zhang, T.-F. Liu, D. Feng, X. Wang, J. Qin and H.-C. Zhou, J. Am. Chem. Soc., 2015, 137, 3177-3180.

24. V. Bon, V. Senkovskyy, I. Senkovska and S. Kaskel, Chem. Commun., 2012, 48, 84078409.

25. A. Schaate, P. Roy, T. Preuße, S. J. Lohmeier, A. Godt and P. Behrens, Chem. Eur. J, 2011, 17, 9320-9325.

26. S. Marx, W. Kleist and A. Baiker, J. Catal., 2011, 281, 76-87.

27. O. Kozachuk, I. Luz, F. X. Llabrés i Xamena, H. Noei, M. Kauer, H. Bauke Albada, E. D. Bloch, B. Marler, Y. Wang, M. Muhler and R. A. Fischer, Angew. Chem., Int. Ed., 2014, 53, 7058-7062.

28. W. Zhang, M. Kauer, O. Halbherr, K. Epp, P. Guo, M. I. Gonzalez, D. J. Xiao, C. Wiktor, F. X. Llabrés i Xamena, C. Wöll, Y. Wang, M. Muhler and R. A. Fischer, Chem. Eur. J, 2016, 22, 14297-14307.

29. E. Plessers, G. Fu, C. Y. X. Tan, D. E. de Vos and M. B. J. Roeffaers, Catalysts, 2016, 6, 104.

30. L. Valenzano, B. Civalleri, S. Chavan, S. Bordiga, M. H. Nilsen, S. Jakobsen, K. P. Lillerud and C. Lamberti, Chem. Mater., 2011, 23, 1700-1718. 
31. V. Bon, I. Senkovska, I. A. Baburin and S. Kaskel, Cryst. Growth Des., 2013, 13, 1231 1237.

32. C. F. de Graauw, J. A. Peters, H. Van Bekkum and J. Huskens, Synthesis, 1994, 10, 10071017.

33. MOPAC2016, HTTP://OpenMOPAC.net/, Version 17.048W, J.J.P. Stewart, Stewart Computational Chemistry, Colorado Springs, CO, USA

34. J. A. Dodge and C. W. Lugar III, Bioorg. Med. Chem. Lett., 1996, 6, 1-2.

35. G.-D. Han, Z.-Y. Lin and C.-P. Yan, Zhongguo Yiyao Gongye Zazhi, 1994, 25, 490-491.

36. T. Ohta, H. Zhang, Y. Torihara, T. Hida and I. Furukawa, Steroids, 1998, 63, 630-632.

37. G. Göndös and J. C. Orr, J. Chem. Soc., Chem. Commun., 1982, 1238-1239.

38. J. C. van der Waal, K. Tan and H. Van Bekkum, Catal. Lett., 1996, 41, 63-67.

39. A. Corma, M. E. Domine, L. Nemeth and S. Valencia, J. Am. Chem. Soc., 2002, 124, 3194-3195. 